\title{
KJCCM
}

\section{Spontaneous Echo Contrast Mistaken for Left Ventricular Thrombus during Venoarterial Extracorporeal Membrane Oxygenation}

\author{
Seok In Lee, So Young Lee, Chang Hyu Choi, Kook Yang Park, and Chul-Hyun Park \\ Department of Thoracic and Cardiovascular Surgery, Gil Medical Center, Gachon University, Incheon, Korea
}

\section{Dear Editor:}

Spontaneous echo contrast (SEC) is often observed in patients with mitral stenosis, atrial fibrillation, cardiomyopathy, or a ventricular aneurysm [1]. SEC is a smoke-like echo density observed on echocardiograms, and is caused by increased red blood cell aggregation during low-flow states. It is also a risk factor of thromboembolism [2]. SEC can be observed in patients with severe ventricular dysfunction receiving venoarterial extracorporeal membrane oxygenation (VA-ECMO). We present a case in which left ventricular-SEC (LV-SEC) was mistaken for a LV thrombus during VA-ECMO for severe LV dysfunction.

A 36-year-old female patient diagnosed with acute fulminant myocarditis was provided VA-ECMO support on hospital day (HD) 1. Briefly, VA-ECMO (RotaFlow; Maquet Inc., Hirrlingen, Germany) was implanted in the right femoral artery (15-French arterial cannula) and the left femoral vein (20-French venous cannula). Her height and body weight are $163 \mathrm{~cm}$ and $52 \mathrm{~kg}$ (body surface area, $1.53 \mathrm{~m}^{2}$ ). VAECMO was initiated with a circuit flow of $3.5 \mathrm{~L} / \mathrm{min}$ (cardiac index, $2.32 \mathrm{~L} / \mathrm{min} / \mathrm{m}^{2}$ ). Her creatine kinase-myocardial band and troponin-I levels at admission were 188.03 $\mathrm{ng} / \mathrm{ml}$ (normal range, 0 to $5 \mathrm{ng} / \mathrm{ml}$ ) and $>50.0 \mathrm{ng} / \mathrm{ml}$ (normal range, 0 to $0.78 \mathrm{ng} / \mathrm{ml}$ ), respectively. Impaired ventricular function (ejection fraction, 22\%) suspected as acute fulminant myocarditis was detected by transthoracic echocardiography (TTE) at admission.

TTE revealed decreased LV function (ejection fraction, 10\%) with mild mitral regurgitation (grade II) immediately after VA-ECMO. Opening of the aortic valve and arterial pulsatility were not observed. Pulmonary edema was aggravated on HD 4. Left atrial (LA) decompression was achieved using a LA catheter (20-French femoral venous cannula) by balloon atrial septostomy through the right femoral
Received on March 31, 2017

Revised on May 26, 2017

Accepted on May 26, 2017

Chul-Hyun Park

Department of Thoracic and Cardiovascular Surgery, Gil Medical Center, Gachon University, 21 Namdong-daero 774beongil, Namdong-gu, Incheon 21565, Korea

Tel: +82-32-460-3666

Fax: +82-32-460-2371

E-mail: cdgpch@gilhospital.com

*No potential conflict of interest relevant to this article was reported.

cc This is an Open Access article distributed under the terms of the Creative Commons Attribution Non-Commercial License (http://creativecommons.org/ licenses/by-nc/4.0/) which permits unrestricted non-commercial use, distribution, and reproduction in any medium, provided the original work is properly cited. Copyright (c) 2017 The Korean Society of Critical Care Medicine 

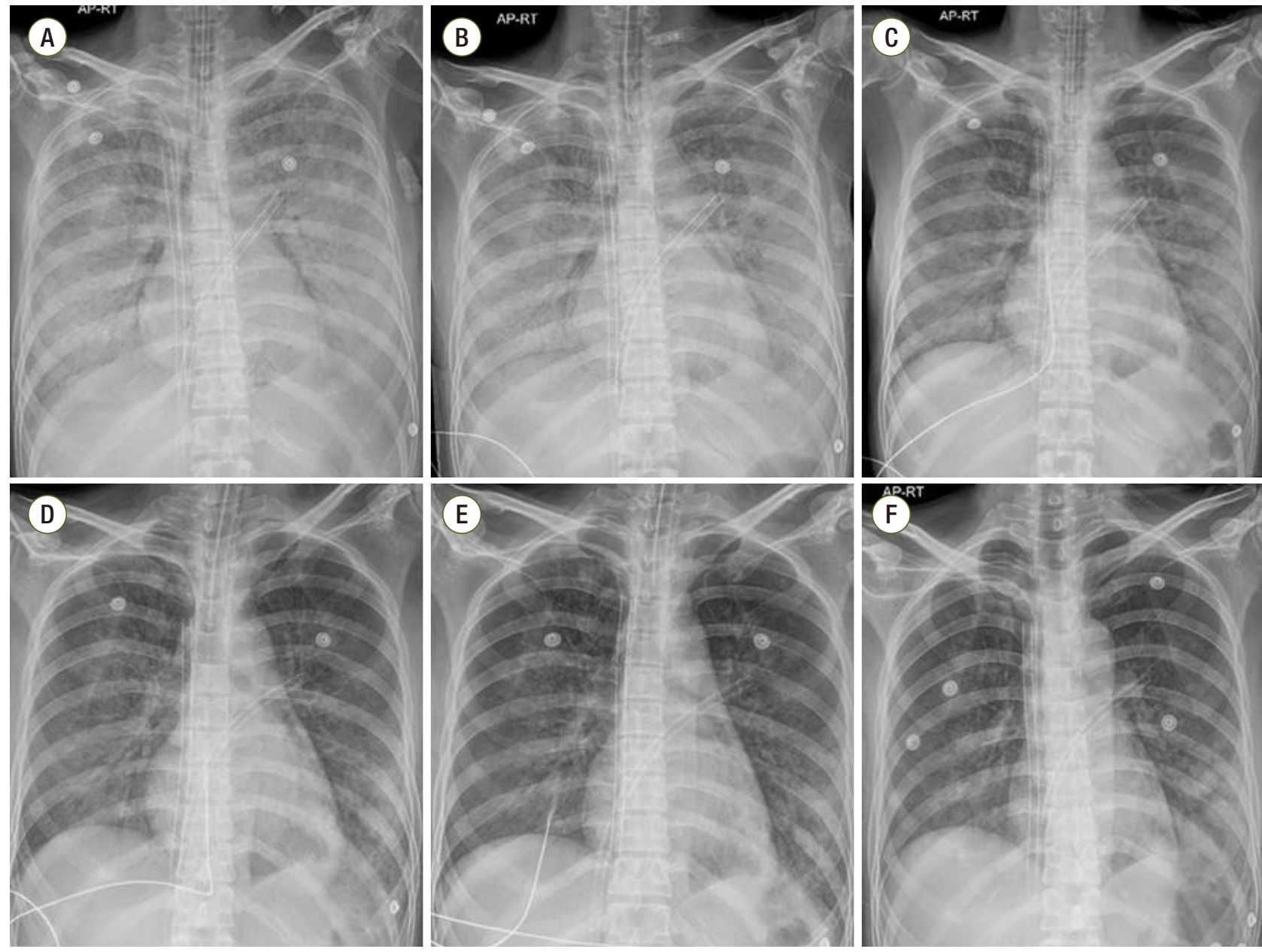

Figure 1. Chest radiographs obtained on hospital days 4 to 9. (A) 4, (B) 5, (C) 6, (D) 7, (E) 8, (F) 9 hospital days.

vein. Pulmonary edema and cardiomegaly improved after LA decompression (Figure 1) and cardiac enzymes levels were reduced (Figure 2).

Input and output were controlled to improve pulmonary edema; about 5,000 $\mathrm{ml}$ volume was removed from HD 5 to 10 . On HD 11, ECMO flow was abruptly reduced from 3.2 to $1.6 \mathrm{~L} / \mathrm{min}$, and hyperechogenic material was detected in the LV using a portable TTE (Figure 3A). In view of the abrupt ECMO flow reduction, we considered LV thrombus, but after infusing normal saline $(500 \mathrm{ml})$ ECMO flow recovered and the hyperechogenic material disappeared (Figure 3B). We realized that LV-SEC was misdiagnosed as LV thrombus. At that time, her activated prothrombin time was 100 seconds, and fortunately there was no evidence of thromboembolic complications. Her cardiac function then recovered and on HD 14 VA-ECMO was weaned without embolic or bleeding complications.

VA-ECMO administered using peripheral cannulation induces retrograde blood flow and increases afterload. If LV dysfunction is severe, the aortic valve may not open against a high afterload and pulsatility may disappear. In such cases, blood stasis and SEC are observed [3]. In the absence of pulsatility, TTE is important to ensure the presence of swirling. Excessive volume removal and LA venting can aggravate SEC because preload is lowered and the LV cavity is emptied [4]. Furthermore, if ECMO flow decreases abruptly, SEC may be mistaken for thrombus. In this context, cautious interpretation of echocardiographic finding based on the clinical situation 


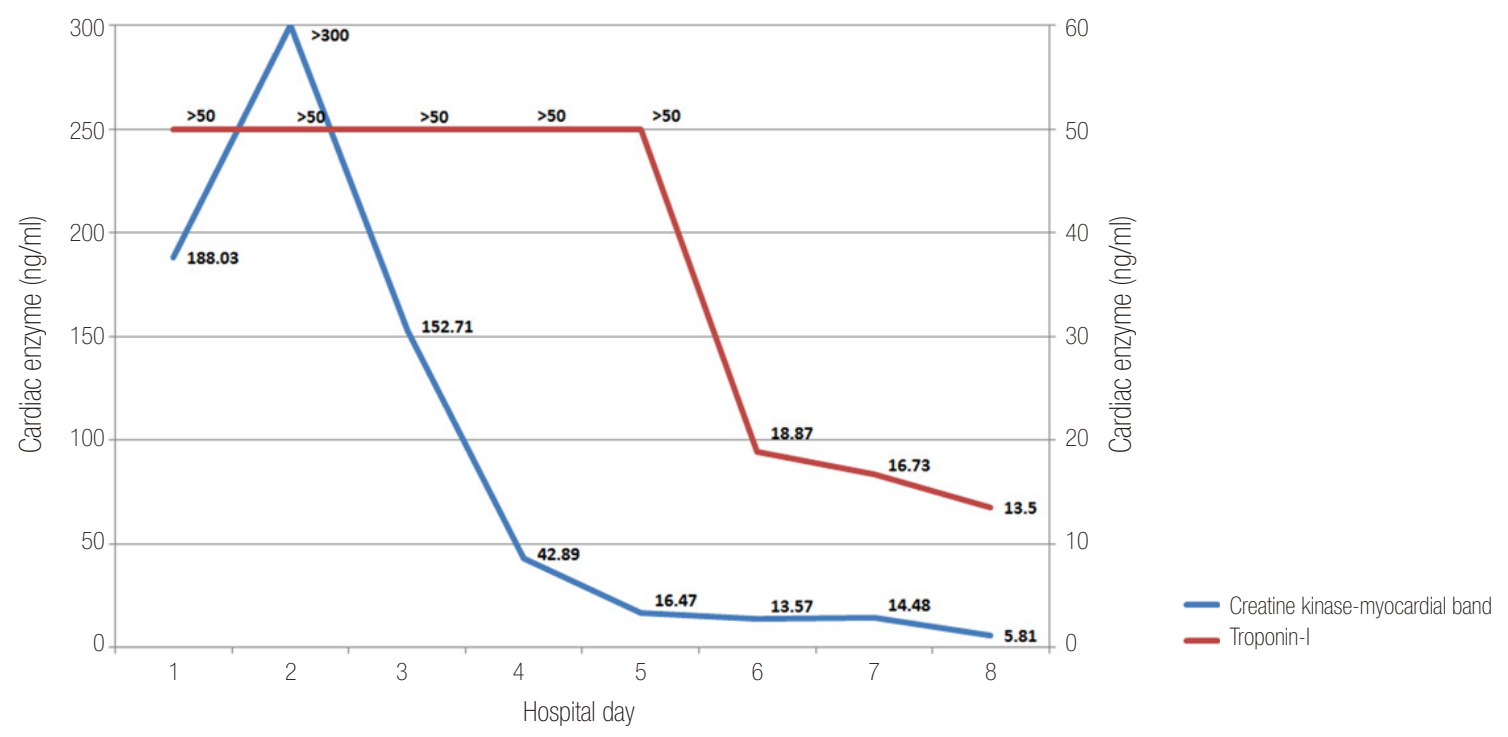

Figure 2. Cardiac enzyme trends.
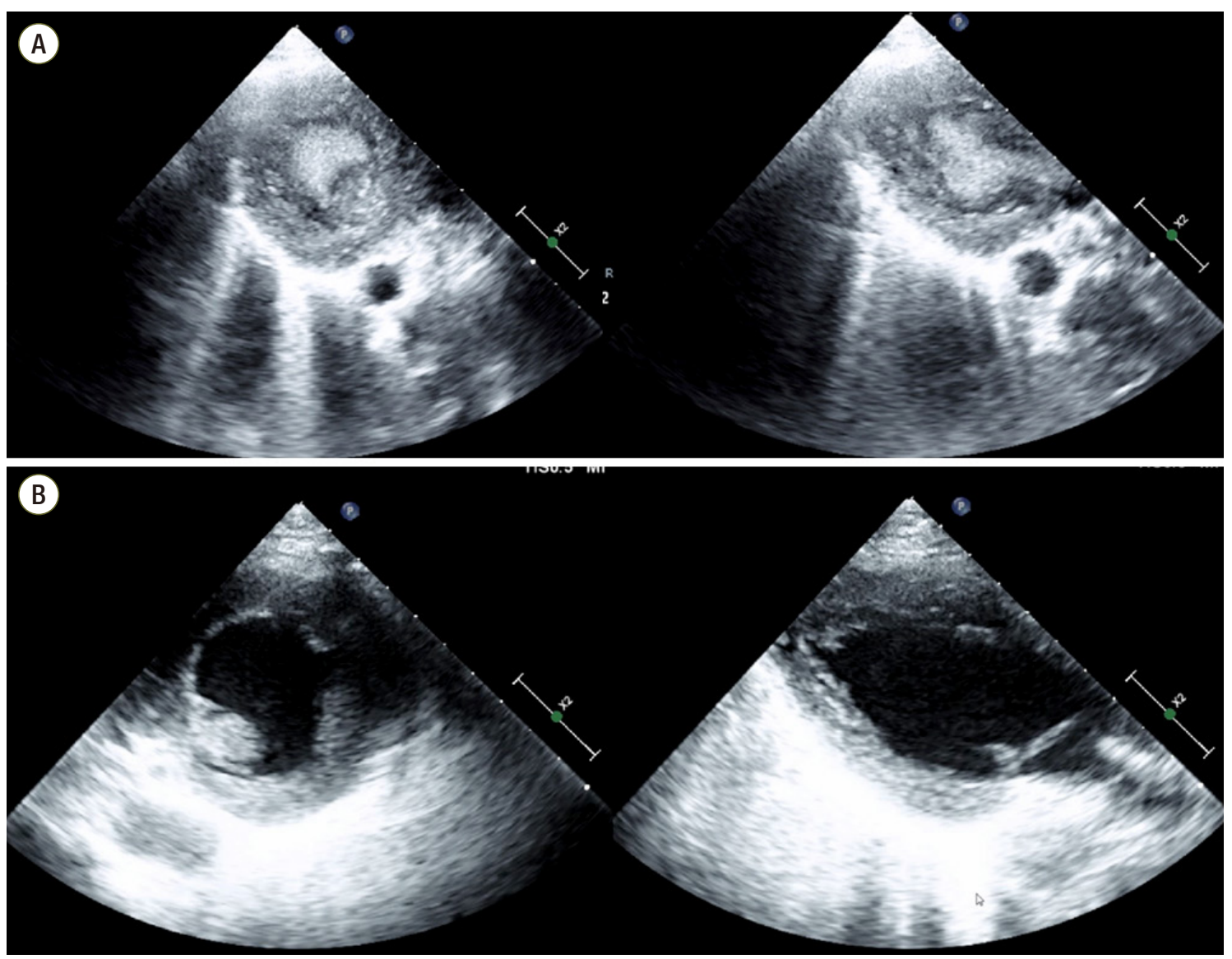

Figure 3. Trans-thoracic echocardiographs obtained on hospital day 11. (A) Spontaneous echo contrast mistaken for left ventricular thrombus (parasternal short and long axis views). (B) Disappeared spontaneous echo contrast after volume replacement (parasternal short and long axis views). 
is important to avoid invasive procedures, such as exploratory cardiac surgery.

It is also important to reduce the afterload for improving the pulsatility. Combining ECMO with intra-aortic balloon pump may have benefit in term of decreasing the afterload [5]. Vasodilator medications, such as nitroprusside, nitroglycerin, calcium channel blockers can reduce the afterload as well. The flow of VA-ECMO should also be adjusted while confirming the perfusion status and mean arterial pressure [6].

Myocarditis is a hypercoagulable state induced by a systemic inflammatory process [7], and thus adequate anticoagulation therapy is required during VA-ECMO to prevent thrombotic complications. In particular, when SEC is observed in a patient with severe LV dysfunction, the risk of these complications increases. In the described case, we performed VA-ECMO for acute fulminant myocarditis with severe LV dysfunction, and followed this with daily echocardiography to identify swirling or SEC. Target activated prothrombin time of heparin therapy was maintained at a high level ( $>70$ seconds) because the aortic valve did not open and swirling was observed. Furthermore, if our patient had not been given inappropriate anticoagulation therapy when SEC was present, complications of thrombus would certainly have occurred.

In conclusion, VA-ECMO in patients with acute fulminant myocarditis and severe LV compromise provides meaningful life support and facilitates the recovery of cardiac function. However, SEC can occur in the absence of pulsatility or aortic valve opening, and severe volume restriction and LA venting might increase the risk of LVSEC by emptied the LV cavity. LV-SEC may be mistaken for thrombus when ECMO flow abruptly reduced, and thus, repeat evaluation using TTE and cautious interpretation of echocardiographic finding are required. On the other hand, in patients with SEC, proper anticoagulation therapy is important to prevent complications of thrombus.
ORCID

Seok In Lee

http://orcid.org/0000-0002-8538-4511

\section{References}

1. Black IW. Spontaneous echo contrast: where there's smoke there's fire. Echocardiography 2000;17:37382.

2. Black IW, Hopkins AP, Lee LC, Walsh WF. Left atrial spontaneous echo contrast: a clinical and echocardiographic analysis. J Am Coll Cardiol 1991;18:398-404

3. Unai S, Nguyen ML, Tanaka D, Gorbachuk N, Marhefka GD, Hirose H, et al. Clinical significance of spontaneous echo contrast on extracorporeal membrane oxygenation. Ann Thorac Surg 2017;103:773-8.

4. Pepi M, Evangelista A, Nihoyannopoulos P, Flachskampf FA, Athanassopoulos G, Colonna P, et al. Recommendations for echocardiography use in the diagnosis and management of cardiac sources of embolism: European Association of Echocardiography (EAE) (a registered branch of the ESC). Eur J Echocardiogr 2010;11:461-76.

5. Ma P, Zhang Z, Song T, Yang Y, Meng G, Zhao J, et al. Combining ECMO with IABP for the treatment of critically ill adult heart failure patients. Heart Lung Circ 2014;23:363-8.

6. Chung M, Shiloh AL, Carlese A. Monitoring of the adult patient on venoarterial extracorporeal membrane oxygenation. ScientificWorldJournal 2014;2014:393258.

7. Atas H, Samadov F, Sunbul M, Cincin A, Delil K, Mutlu B. Two cases of acute myocarditis with multiple intracardiac thrombi: the role of hypercoagulable states. Heart Views 2014;15:22-5. 